\title{
SPECIES COMPOSITION AND \\ ABUNDANCE OF TINTINNIDS AND COPEPODS \\ IN THE PICHAVARAM MANGROVES (SOUTH INDIA)
}

\author{
COMPOSICION DE ESPECIES Y \\ ABUNDANCIA DE TINTINIDOS Y COPEPODOS \\ EN LOS MANGLARES DE PICHAVARAM (SUR DE LA INDIA)
}

\author{
N. Godhantaraman \\ Centre of Advanced Study in Marine Biology \\ Annamalai University \\ Parangipcttai 608502 \\ Tamil Nadu, India
}

Recibido en marzo de 1992; aceptado en junio de 1994

\begin{abstract}
The annual pattern of distribution and abundance of tintinnids and copepods -taking them as the representatives of the major components of microzooplankton (20 to $200 \mu \mathrm{m})$ and mesozooplankton (200 $\mu \mathrm{m}$ to $2 \mathrm{~mm}$ ), respectively - was studied at the Pichavaram mangroves (India). Monthly samplings were made from January to December 1989, covering three stations. For tintinnids, density varied from 60 to $44,990 \mathrm{org} . / \mathrm{m}^{3}$. Agglomerated tintinnids were the dominant form. The maximum abundance $\left(80,740 \mathrm{org} . / \mathrm{m}^{3}\right)$ of copepods was recorded in the freshwater and neritic water mixing zone (station 2) during summer and the lowest $\left(80 \mathrm{org} . / \mathrm{m}^{3}\right)$ in the freshwater zone (station 1). The genera Acartia and Acrocalanus (belonging to the suborder Calanoida), Macrosetella and Euterpina (belonging to the Harpacticoida), and Oithona (belonging to the Cyclopoida) were coincident with the maximum species abundance at station 2. Species like Acartia southwellii and Oithona brevicornis were observed during the lower abundance period. Calanoid and harpacticoid copepods were also dominant at station 3. A significant positive relationship between the abundance of tintinnids and copepods was observed. The seasonal distribution and abundance of tintinnids and copepods are dis-

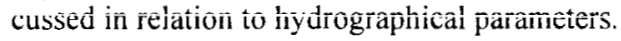

Key words: tintinnids, copepods, South India, distribution, abundance.

\section{RESUMEN}

Se estudió el patrón anual de distribución y abundancia de tintínidos y copépodos - tomados como representantes de los mayores componentes del microzooplancton $(20$ a $200 \mu \mathrm{m})$ y mesozooplancton $(200 \mu \mathrm{m}$ a $2 \mathrm{~mm})$, respectivamente - , en los manglares de Pichavaram (India). Se tomaron muestras de enero a diciembre de 1989, en tres estaciones. La densidad de los tintínidos varió de 60 a 44,990 org. $/ \mathrm{m}^{3}$. Los tintínidos aglomerados fueron la forma dominante. La abundancia máxima de copépodos $\left(80,740 \mathrm{org} . \mathrm{m}^{3}\right)$ se registró en la zona de mezcla de agua dulce y nerítica

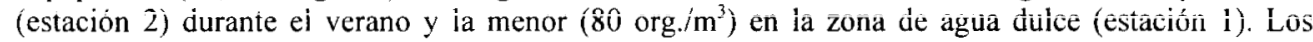
géneros Acartia y Acrocalanus (pertenecientes al suborden Calanoida), Macrosetella y Euterpina (pertenecientes al Harpacticoida) y Oithona (perteneciente al Cyclopoida) coincidieron con la máxima abundancia de especies en la estación 2. Se observaron especies como Acartia southwellii y Oithona 
brevicornis en el periodo de menor abundancia. Los copépodos calanoides y harpacticoides también dominaron en la estación 3. Se observó una notable relación positiva entre la abundancia de tintínidos y copépodos. Se discute la distribución estacional y abundancia de tintínidos y copépodos en relación con los parámetros hidrográficos.

Palabras clave: tintínidos, copépodos, India del Sur, distribución, abundancia.

\section{INTRODUCTION}

The tropics are well known for the wide diversity in species composition, both within the plant and animal kingdoms. Likewise, plankton of the tropics are variegated and change their composition daily (Krishnamurthy, 1962).

The plankton are broadly divisible into the following based on their cell size (Dussart, 1965): ultraplankton $(<2 \mu \mathrm{m})$, nannoplankton (2-20 $\mu \mathrm{m})$. microzooplankton $(20-200 \mu \mathrm{m})$, mesozooplankton $(200 \mu \mathrm{m}-2 \mathrm{~mm})$, megaplankton $(>20 \mathrm{~mm})$.

Among the planktonic organisms, the micro and mesozooplankters play an important connecting role in the pelagic or aquatic food web. They form a vital link for turnover of organic matter and transfer of energy to higher trophic levels of organic production between the primary producers like diatoms and the secondary consumers like fish (Krishnamurthy et al., 19/9). Among microzooplankton, tintinnids are common and dominant in this region. They are the important food source of mesozooplankton.

These copepods graze on the primary producers, which form their staple food. The organisms at tertiary level and at higher trophic levels feed mainly upon zooplankton (Krishnamurthy et al. 1979). Some studies on the annual distribution patterns of copepods in relation to microzooplankton have been made in the Pichavaram mangroves and adjacent biotopes (Krishnamurthy and Santhanam, 1975; Damodara Naidu et al., 1977; Damodara Naidu, 1980) and elsewhere (Beers and Stewart, 1969; Conover. 1982: Capriulo and Carpenter, 1983).

In this study. simultaneous in situ variations in species composition and population of tintinnids and copepods (the major components of microzooplankton and mesozooplankton, respectively) were investigated for a period of

\section{INTRODUCCION}

Es bien conocida la amplia diversidad de los trópicos en cuanto a composición de especies de los reinos vegetal y animal. El plancton de los trópicos es igualmente diverso y cambia diariamente su composición (Krishnamurthy, 1962).

En términos generales, el plancton se divide de acuerdo con el tamaño de su célula (Dussart, 1965): ultraplancton $(<2 \mu \mathrm{m})$, nanoplancton $(2$ a $20 \mu \mathrm{m})$, microzooplancton $(20$ a $200 \mu \mathrm{m})$, mesozooplancton $(200 \mu \mathrm{m}$ a $2 \mathrm{~mm})$, megaplancton ( $>20 \mathrm{~mm}$ ).

Entre los organismos planctónicos, los micro y mesozooplanctónicos representan una función importante de conexión en la red alimentaria pelágica o acuática. Constituyen un enlace vital para la renovación de materia orgánica y la transferencia de energía a niveles tróficos superiores de producción orgánica entre los productores primarios, como las diatomeas, y los consumidores secundarios. como los peces (Krishnamurthy et al., 1979). En el microzooplancton, los tintínidos son comunes y dominantes en esta región. Son la fuente alimentaria importante del mesozooplancton.

Estos copépodos se alimentan de productores primarios, que forman su alimento básico. Los organismos del tercer nivel y niveles tróticos superiores se alimentan principalmente de zooplancton (Krishnamurthy et al., 1979). Se han efectuado algunos estudios sobre los patrones de distribución anual de los copépodos en relación con el milcrozooplancton, en los manglares de Pichavaram y los biotopos adyacentes (Krishnamurthy y Santhanam, 1975; Damodara Naidu et al, 1977: Damodara Naidu. 1980), así como en otros lugares (Beers y Stewart, 1969; Conover, 1982; Capriulo y Carpenter, 1983). 


\section{STUDY AREA}

The present study was carried out at the Pichavaram mangroves located near Parangipettai (Porto Novo), India, on the Bay of Bengal seaboard $\left(11^{\circ} 29^{\prime} \mathrm{N}, 79^{\circ} 45^{\prime} \mathrm{E}\right)$ (Fig. 1). This ecosystem is unique as it is connected with the other aquatic biotopes, such as the estuary, the backwater and the sea. Such a situation promotes mixing of waters of varying salinity, temperature, nutrient concentrations, etc. The mixed waters of the mangroves are very fertile and productive (Sundararaj and Krishnamurthy, 1974), enabling growth of a wide variety of organisms, shellfish and finfish, besides plankton.

In the present study, three ecologically different study sites were chosen; the distance between the stations was about $1 \mathrm{~km}$ (Fig. 1). Station 1, Karithurai, is located in the freshwater part of the mangroves; station 2, Madai, is located in the mixing zone of freshwater and neritic water; station 3, Periyakadavu, is situated in the interior area. The banks of the waterway in this area are lined by the dense mangrove vegetation.

In this tropical region, based on the climatic variations, the year is divisible intu four climatic seasons: monsoon (rainy season), October to December; postmonsoon, January to March; summer, April to June; premonsoon, July to September.

The salinity of the waterways is low during the rainy (monsoon) season and high during the summer season. The average annual precipitation in this area is about $130 \mathrm{~cm}$.

\section{MATERIALS AND METHODS}

Mesozooplankton samples were collected monthly (table 1) using a conical net (mesh size $119 \mu \mathrm{m}$ ), having a mouth arca of $0.25 \mathrm{~m}^{2}$; the net was towed for five minutes to collect the surface zooplankton. A net (mesh size $54 \mu \mathrm{m}$ ) was also towed for five minutes for the collection of microzooplankton during high tide. The net was towed horizontally $(0 \mathrm{~m}$ depth $)$ at the surface water for a distance of about $50 \mathrm{~m}$. To collect a good number of species and estimate the abundance of mesozooplankton (copepods), 2001 of surface water were filtered with the
En este estudio, se investigaron las variaciones simultáneas in situ de la composición de especies y la población de tintínidos y copépodos (los mayores componentes del microzooplancton y mesozooplancton, respectivamente), durante un año, de enero a diciembre de 1989.

\section{AREA DE ESTUDIO}

El presente estudio se llevó a cabo en los manglares de Pichavaram, localizados cerca de Parangipettai (Porto Novo), India, sobre el litoral de la balía de Bengalia (11 $29^{\prime} \mathrm{N}, 79^{\circ} 45^{\prime} \mathrm{E}$ ) (Fig. 1). Este ecosistema es único pues está conectado con otros biotopos acuáticos: estuario, remanso y mar. Tal situación promueve la mezcla de aguas de diferentes salinidades, temperaturas, concentraciones de nutrientes, etc. Las aguas mezcladas de los manglares son muy fértiles y productivas (Sundararaj y Krishnamurthy, 1974). Permiten el crecimiento de una amplia variedad de organismos, crustáceos y peces ue escama, además de plancton.

En la presente investigación, se escogieron tres lugares de estudio diferentes ecológicamente; la distancia entre las estaciones fue de cerca de $1 \mathrm{~km}$ (Fig. 1). La estación 1, Karithurai, se localiza en la parte de agua dulce de los manglares; la estación 2, Madai, se ubica en la zona de mezcla de agua dulce y agua neritica; la estación 3, Periyakadavu, está situada en el área interior. Las orillas del canal de esta área están bordeadas por la densa vegetación del manglar.

En esta región tropical, con base en las variaciones climáticas, el año se divide en cuatro estaciones: monzón, octubre a diciembre (lluvias); postmonzón, enero a marzo; verano, abril a junio; premonzón, julio a septiembre.

Durante la temporada de lluvias (monzón), la salinidad de los canales es baja y durante el verano, alta. El promedio anual de precipitación en esta área es de cerca de $130 \mathrm{~cm}$.

\section{MATERIALES Y METODOS}

Se recolectaron mensualmente muestras de mesozooplancton (tabla I) usandio una red cónica (luz de malla de $119 \mu \mathrm{m}$ ), con un área de boca de $0.25 \mathrm{~m}^{2}$; la red se arrastró durante cinco minutos para recolectar el zooplancton de la su- 


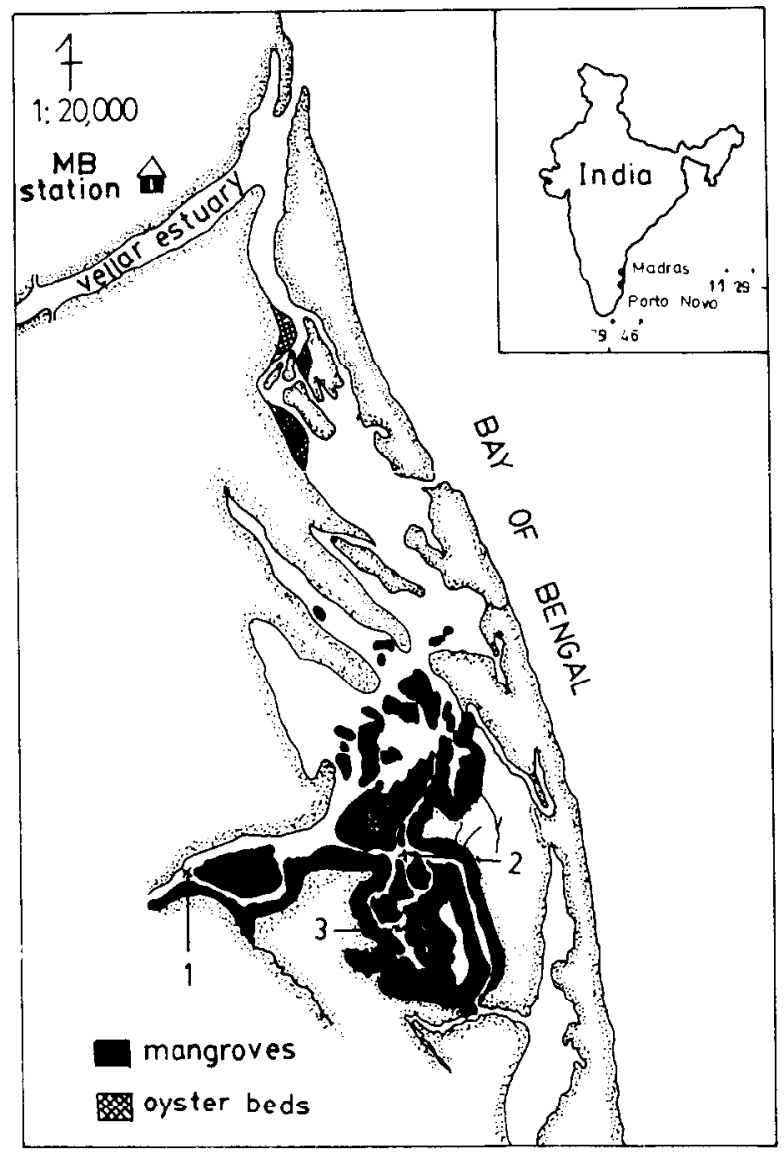

Figure 1. Map showing the study area. Numbers 1-3 indicate the study areas in the Pichavaram mangroves.

Figura 1. Mapa del área de estudio. Los números 1 a 3 indican las áreas de estudio en los manglares de Pichavaram.

help of a 20-I bucket (10 times). When compared to mesozooplankton, microzooplankton cell size is much smaller and there is a considerable chance of missing a few smaller-sized forms through the net. Hence, to nbtain reasonable values in the tintinnid abundance the sedimentation technique was used, following the method of Shukhanova (1978). According to this method, one-litre surface water samples were collected using polyethylene bottles and fixed with $5 \%$ neutralized formalin on board. The bottles were kept undisturbed for 48 hours till complete sedimentation; microzooplankton settled on the bottom and was used for numerical counts, using Utermöhl's inverted plankton perficie. También se arrastró cinco minutos una red (luz de malla de $54 \mu \mathrm{m}$ ) para recolectar microzooplancton durante la marea alta. La red se arrastró horizontalmente sobre la superficie del agua $(0 \mathrm{~m}$ de profundidad $)$. una distancia de aproximadamente $50 \mathrm{~m}$. Con el fin de recolectar un buen número de especies y estimar la abundancia de! mesozooplancton (copépodos), se filtraron $200 \mathrm{l}$ de agua de la superficie con la ayuda de una cubeta de 201 (diez veces). El tamaño de célula del microzooplancton comparado con el del mesozooplancton, resulta mucho menor y es considerable la posibilidad de perder algunas tormas de las tallas menores, a través de la red. Por tanto, para obtener va- 
Table 1. Month, day and hour of sampling,

Tabla 1. Mes, día y hora de muestreo.

\begin{tabular}{lclcrc}
\hline Month & Date & Day & Station 1 & Station 2 & Station 3 \\
\hline January & $2 / 01 / 89$ & Monday & $8: 30$ a.m. & $9: 20$ a.m. & $10: 20$ a.m. \\
February & $2 / 02 / 89$ & Thursday & $7: 45 \mathrm{a} . \mathrm{m}$. & $8: 35 \mathrm{a} . \mathrm{m}$. & $9: 45 \mathrm{a} . \mathrm{m}$. \\
March & $4 / 03 / 89$ & Satuidday & $3: 15 \mathrm{p} . \mathrm{m}$. & $4: 10 \mathrm{p} . \mathrm{m}$. & $5: 20 \overline{\mathrm{p}} . \mathrm{m}$. \\
April & $3 / 04 / 89$ & Monday & $8: 15 \mathrm{a} . \mathrm{m}$. & $9: 20 \mathrm{a} . \mathrm{m}$. & $10: 10 \mathrm{a} . \mathrm{m}$. \\
Mayy & $2 / 05 / 89$ & Tuesday & $9: 45 \mathrm{a} . \mathrm{m}$. & $10: 40 \mathrm{a} . \mathrm{m}$. & $11: 40 \mathrm{a} . \mathrm{m}$. \\
June & $3 / 06 / 89$ & Saturday & $10: 40 \mathrm{a} . \mathrm{m}$. & $11: 35 \mathrm{a} . \mathrm{m}$. & $12: 30 \mathrm{a} . \mathrm{m}$. \\
July & $3 / 07 / 89$ & Monday & $7: 15 \mathrm{a} . \mathrm{m}$. & $8: 20 \mathrm{a} . \mathrm{m}$. & $9: 15 \mathrm{a} . \mathrm{m}$. \\
August & $2 / 08 / 89$ & Wednesday & $8: 45 \mathrm{a} . \mathrm{m}$. & $9: 50 \mathrm{a} . \mathrm{m}$. & $10: 40 \mathrm{a} . \mathrm{m}$. \\
September & $6 / 09 / 89$ & Wednesday & $8: 45 \mathrm{a} . \mathrm{m}$. & $9: 35 \mathrm{a} . \mathrm{m}$. & $10: 20 \mathrm{a} . \mathrm{m}$. \\
October & $4 / 10 / 89$ & Wednesday & $9: 40 \mathrm{a} . \mathrm{m}$. & $10: 35 \mathrm{a} . \mathrm{m}$. & $11: 20 \mathrm{a} . \mathrm{m}$. \\
November & $3 / 11 / 89$ & Friday & $8: 45 \mathrm{p} . \mathrm{m}$. & $9: 35 \mathrm{a} . \mathrm{m}$. & $10.20 \mathrm{a} . \mathrm{m}$. \\
December & $30 / 12 / 89$ & Saturday & $2: 30 \mathrm{p} . \mathrm{m}$. & $3: 20 \mathrm{p} . \mathrm{m}$. & $4: 20 \mathrm{p} . \mathrm{m}$. \\
\hline
\end{tabular}

microscope (Utermöhl, 1958). A water sample was also collected for the measurement of hydrographical parameters such as temperature, salinity and dissolved oxygen. Water temperature was measured using a standard Celsius thermometer. Salinity was estimated by the silver nitrate titration method and dissolved oxygen by the modified Winkler method as described by Strickland and Parsons (1972).

\section{RESULTS}

\section{Environmental parameters}

Environmental parameters showed wide seasonal variations. Highest temperature and salinity were recorded during the summer and lowest during the monsoon season (Figs. 2, 3). The minimum and maximum monthly temperature were $24.5^{\circ} \mathrm{C}$ (December) and $35.5^{\circ} \mathrm{C}$ (June), respectively. Salinity varied widely, ranging from $3.9 \mathrm{ppt}$ (September) to $35.5 \mathrm{ppt}$ (June). The dissolved oxygen values were high $(6.1 \mathrm{~m} / \mathrm{l})$ during the monsoon (December) and low $(3.62 \mathrm{ml} / \mathrm{l})$ during the summer (May) season (Fig. 4). Season-wise observation of dissolved oxygen showed an inverse trend against temperature and salinity. lores razonables de la abundancia de tintínidos, sc usó la técnica de sedimentación, con el métudo de Shukhanova (1978). De acuerdo con este método, se recolectaron muestras de un litro de agua superficial, usando botellas de polietileno y se fijaron con formalina neutralizada, a bordo. Las botellas se mantuvieron quietas por 48 horas, hasta que se completó la sedimentación. El microzooplancton se asentó en el fondo y fue utilizado para el conteo numérico, mediante el microscopio invertido para plancton de Utermöhl (1958). Se recolectó también una muestra de agua para medir parámetros hidrográficos como temperatura, salinidad $\mathrm{y}$ oxigeno disuelto. La temperatura del agua se midió con un termómetro estándar de grados Celsios. La salinidad se estimó con el método de titulación con nitrato de plata, y el oxígeno disuelto con el método de Winkler, modificado tal como lo describen Strickland y Parsons (1972).

\section{RESULTADOS}

\section{Parámetros ambientales}

Los parámetros ambientales muestran amplias variaciones estacionales. Las mayores 


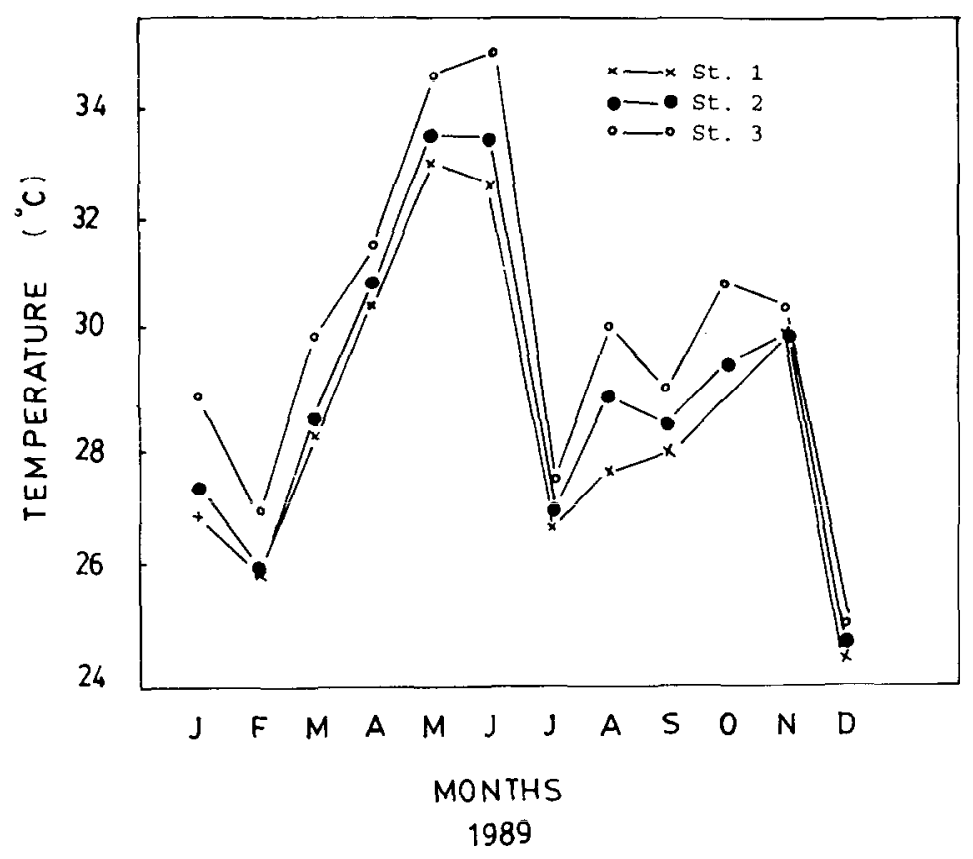

Figure 2. Monthly surface water temperature $\left({ }^{\circ} \mathrm{C}\right)$.

Figura 2. Temperatura $\left({ }^{\circ} \mathrm{C}\right)$ del agua superficial, por mes.

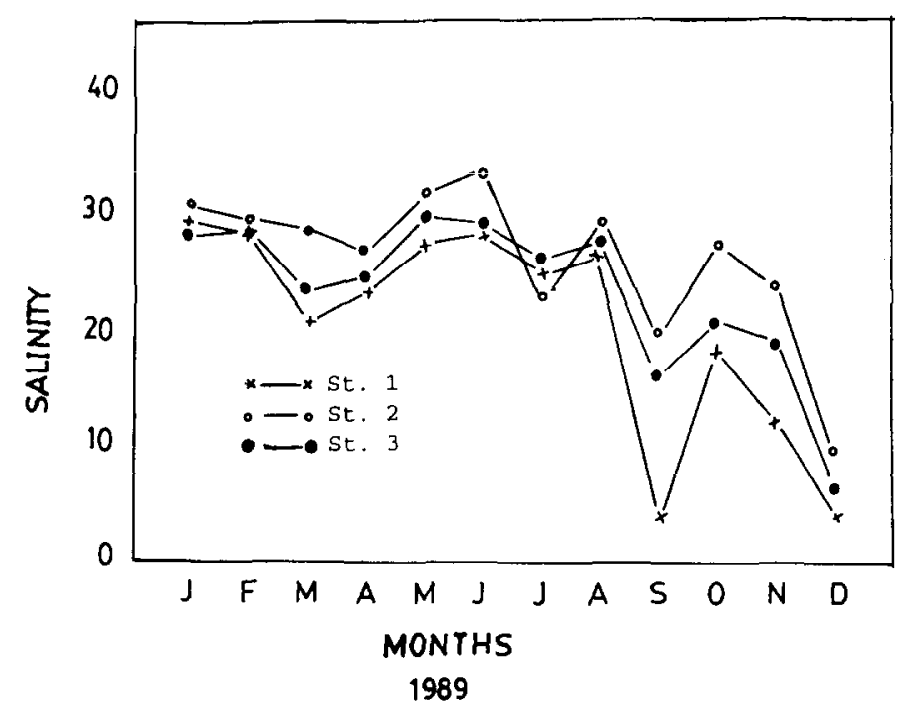

Figure 3. Monthly surface water salinity (ppt).

Figura 3. Salinidad (ppm) del agua superficial, por mes. 
Species composition and abundance of tintinnids

$A$ total of 22 species of tintinnids (Protozoa: Ciliata) were recorded from the waterways of the mangroves. The tintinnids are grouped primarily into agglomerated and non-agglomerated forms. Among these, agglomerated tintinnids constituted major part (18 species) of the total species and were also found to be very important contributors, both qualitatively and quantitatively. These 18 species (agglomerated forms) belong to the genera Tintinnopsis, Tintinnidium, Leptrotintinnus and Codonellopsis. The non-agglomerated forms comprised four species with two genera, Favella and Eutintinnus. Tables 2-4 give the distribution of agglomerated and non-agglomerated forms at all three stations. The distribution of tintinnids showed less variations among stations. Species like Favella brevis, F. philippinensis, Tintinnopsis cylindrica and $T$. beroidea, occurred commonly during the present study. Species like Eutintinnus tenuis and E. lusus-undae occurred only during the monsoon season.

Tintinnid abundance displayed impressive spatial and temporal variations. Figure 5 shows the seasonal abundance of tintinnids. Their seasonal abundance varied, on average, from 3,580 to $17,670 \mathrm{org} . / \mathrm{m}^{3}, 4,480$ to $73,540 \mathrm{org} . / \mathrm{m}^{3}$ and 1,690 to 51,450 org. $/ \mathrm{m}^{3}$ at stations 1,2 and 3 during monsoon and summer, respectively. The highest peak of abundance $\left(44,990 \mathrm{org} . \mathrm{m}^{3}\right)$ was recorded during Iune at station 2. Tintinnopsis cylindrica, $T$. mortensenii and T. tubulo$s a$ were responsible for this peak abundance. The minimum abundance $\left(60 \mathrm{org} . / \mathrm{m}^{3}\right)$ was recorded during September at station 1, with the occurrence of only Tintinnopsis Lohmanni and Favella brevis. The genus Tintinnopsis ranked first in ail seasons during the present study. In general, maximum and minimum abundance of tintinnids were recorded during the summer and monsoon at stations 2 and 3 , respectively.

\section{Species composition and abundance of copepods}

Tables 5-7 show the species occurrence at different stations. A total of 20 species of co- temperaturas y salinidades se registraron durante el verano y las menores, durante las estaciones monzónicas (Figs. 2, 3). Las temperaturas mínima y máxima mensuales fueron $24.5^{\circ} \mathrm{C}$ (diciembre) y $35.5^{\circ} \mathrm{C}$ (junio), respectivamente. La salinidad varió ampliamente, en un intervalo de $3.9 \mathrm{ppm}$ (septiembre) a 35.5 ppm (junio). Los valores de oxígeno disuelto fueron altos $(6.1 \mathrm{ml} / \mathrm{l})$ durante el monzón (diciembre) y bajos (3.62 Inl/l) durante el verano (mayo) (Fig. 4). En el transcursode las estaciones, el oxígeno disuelto mostró una tendencia inversa contra temperatura y salinidad.

\section{Composición de especies y abundancia de tintínidos}

En los canales de los manglares, se registró un total de 22 especies de tintínidos (Protozoa: Ciliata). Los tintínidos se agrupan principalmente en las formas aglomerada y no aglomerada. Los tintínidos aglomerados constituyeron la mayor parte (18 especies) del total de especies y se encontró además que son contribuyentes importantes tanto cualitativa como cuantitativamente. Estas 18 especies (formas ag!omeradas) pertenecen a los géneros Tintinnopsis, Tintinnidium, Leptrotintinnus y Codonellopsis. Las formas no aglomeradas comprendieron cuatro especies con dos géneros, Favella y Eutintinnus. En las tablas 2 a 4, se da la distribución de las formas aglomeradas y no aglomeradas, en las tres estaciones. La distribución de tintínidos muestra menos diferencias entre las estaciones. I as especies como Favella brevis, $F$. philippinensis, Tintinnopsis cylindrica y $T$. beroidea, se encontraron comúnmente, durante el presente estudio. Las especies como Eutintinnus tenuis y E. lusus-undae estuvieron presentes sólo durante la estación del monzón.

La abundancia de tintínidos tuvo variaciones espaciales y temporales impresionantes. La Fig. 5 muestra la abundancia estacional de tintínidos. En promedio, su abundancia varió de 3,580 a 17,670 org. $/ \mathrm{m}^{3}$ en la estación 1 , de 44,990 a 73,540 org. $/ \mathrm{m}^{3}$ en la estación $2 \mathrm{y}$ de 690 a 51,450 org. $/ \mathrm{m}^{3}$ en la estación 3 , durante el monzón y el verano. El mayor pico de abundancia $\left(44,990 \mathrm{org} . / \mathrm{m}^{3}\right)$ se registró durante junio, en la estación 2. Tintinnopsis cylindrica, $T$. mortensenii y $T$. tubulosa fueron las respon- 


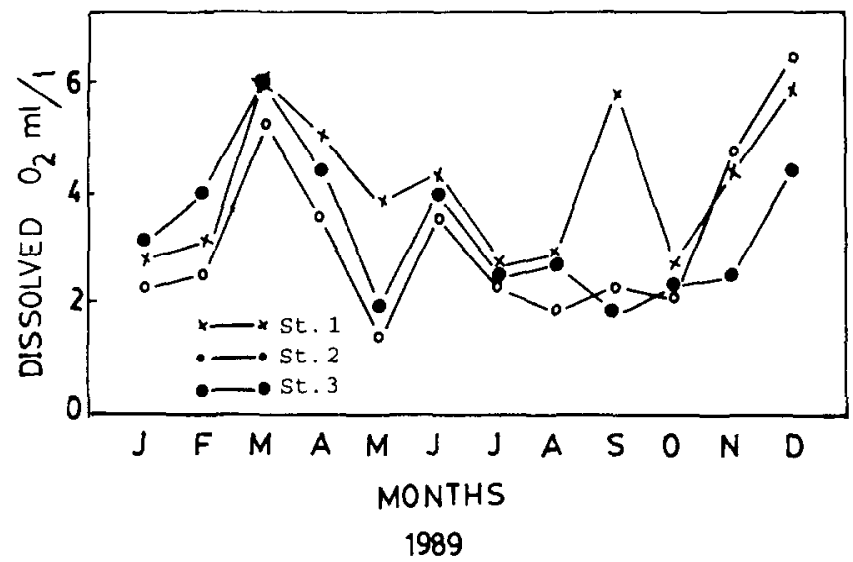

Figure 4. Monthly surface water dissolved oxygen $(\mathrm{ml} / \mathrm{l})$.

Figura 4. Oxígeno disuelto $(\mathrm{ml} / \mathrm{l})$ en el agua superficial, por mes.

\section{Tin-Tintinnids Cop-Copepods}
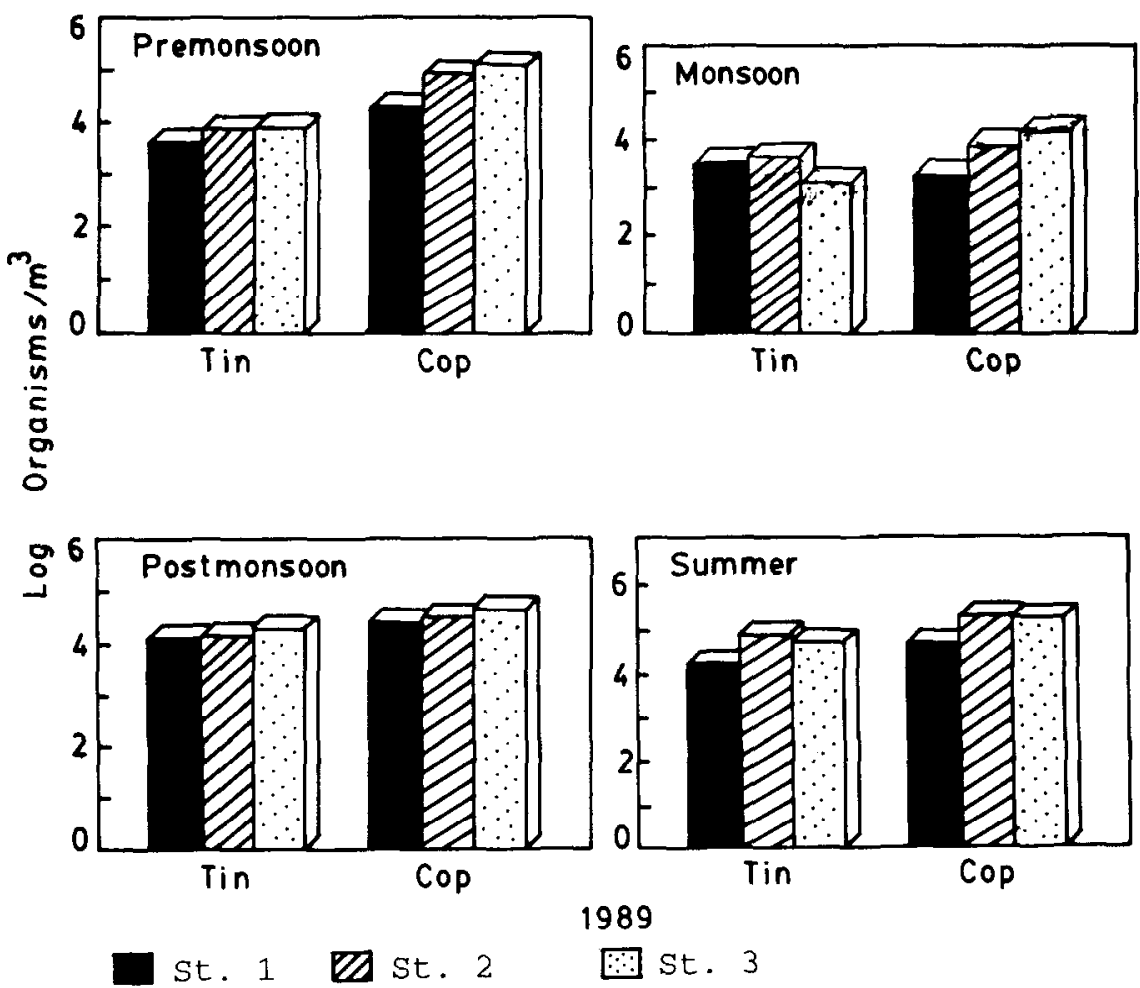

Figure 5. Mean seasonal values of the abundance of tintinnids and copepods at stations 1, 2 and 3. Figura 5. Valores promedio mensuales de abundancia estacional de tintínidos y copépodos, en las estaciones 1,2 y 3 . 
Table 2. Abundance of tintinnids at station $1\left(10^{3} \mathrm{org} / \mathrm{m}^{3}\right)$

Tabla 2. Abundancia de tintinidos en la estación $1\left(10^{7} \mathrm{org} / \mathrm{m}^{3}\right)$.

\begin{tabular}{|c|c|c|c|c|c|c|c|c|c|c|c|c|}
\hline Species & Jan. & Feb. & Mar. & Apr. & May & Jun. & Jul. & Aug. & Sep. & Oct. & Nov. & Dec. \\
\hline \multicolumn{13}{|l|}{ Agglomerated tintinnids } \\
\hline Tintinnidium incertum & 1.03 & 0.69 & 2.44 & - & - & - & - & - & - & - & - & 0.03 \\
\hline Tintinnidium prinitivam & - & 1.84 & - & - & - & - & - & - & - & - & - & - \\
\hline Leprotintinnus simplex & - & 0.03 & - & - & - & - & - & - & - & 0.32 & - & - \\
\hline Tintinnopsis bermudensis & - & - & - & - & - & 1.94 & 0.34 & - & - & - & - & - \\
\hline Tintinnopsis beroidea & - & - & 0.46 & - & - & 0.02 & - & - & - & - & 0.13 & - \\
\hline Tintinnopsis cylindrica & - & 0.34 & 2.70 & - & 0.94 & 2.44 & 0.43 & 0.54 & - & - & - & 0.03 \\
\hline Tintinnopsis directa & - & 0.54 & - & - & 1.03 & - & - & 0.63 & - & 0.39 & - & - \\
\hline Tintinnopsis glans & - & - & - & - & 0.18 & - & 0.06 & - & - & - & - & - \\
\hline Tintinnopsis gracilis & 0.97 & - & - & - & - & - & - & 0.03 & - & - & - & - \\
\hline Tintinnopsis kofoidi & - & - & 0.03 & 0.19 & - & 0.48 & - & - & - & - & - & - \\
\hline Tintinnopsis lohmanni & - & 0.02 & - & - & - & - & 0.59 & - & 0.03 & - & - & - \\
\hline Tintinnopsis minuta & - & - & - & - & - & - & - & 1.03 & - & - & - & - \\
\hline Tintinnopsis mortensenii & - & - & - & 1.95 & 1.84 & - & - & - & - & - & - & - \\
\hline Tintinnopsis nucula & - & - & 0.19 & - & - & - & - & - & - & 0.34 & - & - \\
\hline Tintinnopsis radix & - & - & - & - & - & - & - & 0.01 & - & - & 0.03 & - \\
\hline Tintinnopsis iubulosa & - & - & 0.17 & - & 1.34 & 0.34 & - & - & $=$ & $=$ & $=$ & $=$ \\
\hline Tintinnopsis tocantinensis & - & - & - & - & - & - & - & - & - & - & - & - \\
\hline Codonellopsis ostenfeldii & 0.57 & 0.11 & 0.34 & - & 0.15 & - & - & - & - & - & - & - \\
\hline \multicolumn{13}{|c|}{ Non-agglomerated tintinnids } \\
\hline Favella brevis & - & 0.32 & - & - & 0.55 & 4.17 & - & - & 0.03 & - & 0.18 & 0.05 \\
\hline Favella philippinensis & 0.17 & 0.04 & 0.20 & - & 0.11 & - & 0.05 & 0.20 & - & - & 0.69 & - \\
\hline Eutintinnus tenuis & - & - & - & - & - & - & - & - & - & 0.20 & - & 0.14 \\
\hline Eutintinnus lusus & - & - & - & - & - & - & - & - & - & 1.05 & - & $\cdot$ \\
\hline Total & 2.74 & 3.93 & 6.53 & 2.14 & 6.14 & 9.39 & 1.47 & 2.44 & 0.06 & 2.30 & 1.03 & 0.25 \\
\hline
\end{tabular}


Table 3. Abundance of tintinnids at station $2\left(10^{3} \mathrm{org} . / \mathrm{m}^{3}\right)$.

Tabla 3. Abundancia de tintínidos en la estación $2\left(10^{3} \mathrm{org} . / \mathrm{m}^{3}\right)$.

\begin{tabular}{|c|c|c|c|c|c|c|c|c|c|c|c|c|}
\hline Species & Jan. & Feb. & Mar. & Apr. & May & Jun. & Jul. & Aug. & Sep. & Oct. & Nov. & Dec. \\
\hline \multicolumn{13}{|l|}{ Agglomerated tintinnids } \\
\hline Tintinnidium incertum & 0.32 & - & 1.06 & - & - & - & - & - & - & - & - & - \\
\hline Tintinnidium primitivum & - & 0.25 & 0.82 & - & - & 0.03 & - & - & 0.32 & - & 0.02 & - \\
\hline Leprotintinnus simplex & 0.04 & 0.25 & - & - & - & - & 0.03 & - & - & 2.79 & - & 0.03 \\
\hline Tintinnopsis bermudensis & - & - & 0.32 & - & 0.15 & 0.02 & - & 0.06 & 0.19 & - & - & - \\
\hline Tintinnopsis beroidea & 0.03 & 0.93 & - & - & - & - & 0.06 & 0.24 & - & - & - & - \\
\hline Tintinnopsis cylindrica & - & - & 3.41 & - & - & 32.42 & - & 0.19 & 0.13 & 0.13 & 0.04 & 0.03 \\
\hline Tintinnopsis directa & - & 0.14 & - & - & - & - & 0.03 & 0.16 & 0.27 & - & - & - \\
\hline Tintinnopsis glans & - & - & 0.63 & - & 2.47 & - & 0.27 & - & - & 0.06 & - & - \\
\hline Tintinnopsis gracilis & 0.37 & - & - & - & - & 0.77 & - & 0.01 & 0.33 & - & - & 0.04 \\
\hline Tintinnopsis kofoidi & - & - & - & 0.93 & - & - & - & - & - & 0.02 & - & - \\
\hline Tintinnopsis lohmanni & - & 0.02 & - & - & - & 2.43 & 1.03 & 0.02 & 0.12 & - & - & - \\
\hline Tintinnopsis minuta & - & - & - & - & - & - & 0.75 & - & 0.03 & - & - & 0.06 \\
\hline Tintinnopsis mortensenii & - & - & - & 0.10 & 0.32 & 4.32 & - & 0.03 & - & - & - & - \\
\hline Tintinnopsis nucula & 0.87 & - & 0.09 & - & - & - & 0.67 & - & 0.07 & 0.03 & - & - \\
\hline Tintinnopsis radix & - & - & - & - & - & - & - & 0.03 & - & 0.10 & - & - \\
\hline Tintinnopsis tubulosa & - & 0.19 & - & - & 5.42 & 3.97 & - & 0.27 & - & - & - & - \\
\hline Tintinnopsis tocantinensis & - & - & - & - & 1.82 & - & 0.43 & - & - & 0.02 & - & - \\
\hline Codonellopsis ostenfeldii & - & - & 1.75 & - & - & 0.39 & - & - & - & - & - & - \\
\hline \multicolumn{13}{|c|}{ Non-agglomerated tintinnids } \\
\hline Favella brevis & 0.12 & 2.34 & - & - & - & 0.29 & 0.13 & 0.02 & 0.59 & 0.03 & 0.03 & - \\
\hline Favella philippinensis & - & - & 0.03 & - & 17.43 & 0.35 & 0.03 & 0.03 & 0.13 & 0.62 & - & - \\
\hline Eutintinnus tenuis & - & - & $\cdot$ & - & - & - & - & - & - & - & 0.05 & 0.13 \\
\hline Eutintinnus lusus & - & - & - & - & - & - & - & - & - & 0.19 & 0.02 & 0.04 \\
\hline Total & 1.75 & 4.12 & 8.11 & 1.03 & 27.61 & 44.9 & 3.43 & 1.06 & 2.18 & 3.99 & 0.16 & 0.33 \\
\hline
\end{tabular}


Table 4. Abundance of tintinnids at station $3\left(10^{3} \mathrm{org} . / \mathrm{m}^{3}\right)$.

Tabla 4. Abundancia de tintinidos en la estación $3\left(10^{3} \mathrm{org} . / \mathrm{m}^{3}\right)$.

\begin{tabular}{|c|c|c|c|c|c|c|c|c|c|c|c|c|}
\hline Species & Jan. & Feb. & Mar. & Apr. & May & Jun. & Jul. & Aug. & Sep. & Oct. & Nov. & Dec. \\
\hline \multicolumn{13}{|l|}{ Agglomerated tintinnids } \\
\hline Tintinnidium incertum & 0.13 & - & 3.42 & - & - & - & - & - & - & - & 0.05 & - \\
\hline Tintinnidium primitivum & - & 3.42 & 1.35 & - & 0.05 & - & - & 0.03 & - & 0.03 & - & 0.03 \\
\hline Leprotintinnus simplex & - & - & - & - & - & 0.02 & - & - & - & 0.13 & 0.26 & - \\
\hline Tintinnopsis bermudensis & - & 0.03 & - & - & - & - & $\cdot$ & - & 2.42 & - & - & - \\
\hline Tintinnopsis beroidea & - & 0.08 & 0.79 & - & 3.43 & 0.54 & - & 0.07 & - & - & - & 0.02 \\
\hline Tintinnopsis cylindrica & 0.54 & - & 0.69 & 1.92 & 2.42 & 2.95 & $\cdot$ & - & - & - & - & - \\
\hline Tintinnopsis directa & - & 0.16 & 0.97 & - & - & - & - & 0.02 & - & 0.01 & - & 0.07 \\
\hline Tintinnopsis glans & 0.03 & - & 0.07 & 1.03 & 4.75 & - & 0.32 & - & - & - & 0.11 & - \\
\hline Tintinnopsis gracilis & - & 0.04 & - & - & - & 0.05 & 0.55 & 0.33 & 0.03 & - & - & - \\
\hline Tintinnopsis kofoidi & - & - & - & 0.06 & 1.24 & 4.22 & - & - & - & - & - & - \\
\hline Tintinnopsis lohmanni & 0.03 & - & - & - & - & 0.08 & 0.92 & - & - & - & $\cdot$ & - \\
\hline Tintinnopsis minuta & - & - & 0.32 & - & 0.03 & - & 0.27 & 0.37 & 0.03 & - & - & - \\
\hline Tintinnopsis mortensenii & - & - & - & 0.36 & - & 13.42 & - & - & - & - & - & - \\
\hline Tintinnopsis nucula & - & 2.24 & - & - & 0.02 & - & - & 0.19 & - & 0.04 & - & - \\
\hline Tintinnopsis radix & - & - & - & - & - & - & - & - & 0.07 & - & 0.32 & - \\
\hline Tintinnopsis tubulosa & - & - & 0.54 & 0.07 & - & 0.73 & 0.03 & 0.02 & 0.01 & - & - & - \\
\hline Tintinnopsis tocantinensis & - & - & - & 0.48 & 5.42 & 0.69 & - & - & - & 0.03 & - & - \\
\hline Codonellopsis ostenfeldii & - & - & 0.59 & - & - & - & 0.07 & - & - & - & 0.02 & - \\
\hline \multicolumn{13}{|c|}{ Non-agglomerated tintinnids } \\
\hline Favella brevis & 0.36 & - & 0.06 & 0.03 & - & 0.60 & - & 0.02 & 0.49 & 0.03 & 0.06 & 0.04 \\
\hline Favella philippinensis & $\cdot$ & 0.03 & 4.27 & - & 3.42 & 3.42 & 0.79 & - & - & - & 0.03 & - \\
\hline Eutintinnus tenuis & - & - & - & - & - & - & - & - & - & 0.06 & - & 0.03 \\
\hline Eutintinnus lusus & - & - & - & - & - & - & - & - & - & 0.13 & - & 0.19 \\
\hline Total & 1.09 & 6.00 & 13.07 & 3.95 & 20.78 & 26.72 & 2.95 & 1.05 & 3.05 & 0.46 & 0.85 & 0.38 \\
\hline
\end{tabular}


pepods were recorded, belonging to 13 genera and three major suborders: Calanoida (12 spp.), Harpacticoida (3 spp.) and Cyclopoida (5 spp.). The maximum and minimum numbers of copepods were found at station 2 in May and at station 1 in September, respectively. The genera Acartia, Acrocalanus and Centropages, belonging to calanoids, and Euterpina and Oithona, belonging respectively to harpacticoids and cyclopoids, were the dominant forms. Four species were common to all three stations: Acartia southwellii, Centropages orsinii. Euterpina acutifrons and Oithona rigida. The species Acartia danae, Oithona similis, Corycaeus catus and Labidocera acuta occurred only in the freshwater-neritic mixing zone.

Copepod abundance showed spatial and temporal variations. Figure 5 shows the seasonal abundance of copepods from the three different stations. Their seasonal abundance varied, on average, from 2,280 to $46,830 \mathrm{org} . / \mathrm{m}^{3}$, 7,490 to 180,370 org. $/ \mathrm{m}^{3}$ and 18,930 to $162,180 \mathrm{org} . / \mathrm{m}^{3}$ at stations 1,2 and 3 during the monsoon and summer, respectively. In general, species like Acartia danae, A. southwellii, Acrocalanus longicornis, Pseudodiaptomus serricaudatus, Centropages orsinil, Macrosetella gracilis, Microsetella rosea, Euterpina acutifrons, Oithona rigida, $O$. brevicornis and Corycaeus danae were more in terms of numerical abundance during the present observation.

Species like Acartia southvellii, A. danae, Acrocalanus longicornis, Macrosetella gracilis, Euterpina acutifrons and Oithona similis were responsible for this maximum abundance. Only Acartia southwellii and Oithona brevicornis occurred during the minimum abundance.

\section{Correlation coefficient}

Significant positive correlation was observed between the monthly abundance of copepods and tintinnids at all three stations. Table 8 shows a highly significant correlation between the monthly abundance of tintinnids and that of copepods at all three stations. It might show their food web relationship in this ecosystem. sables de este pico de abundancia. La mínima abundancia ( $60 \mathrm{org} . / \mathrm{m}^{3}$ ) se registró durante septiembre, en la estación 1, sólo con la presencia de Tintinnopsis lohmanni y Favella brevis. E1 género Tintinnopsis se situó en el primer lugar en todas las estaciones del año durante el presente estudio. En general, las abundancias máxima y mínima de tintínidos se registraron durante el verano y el monzón en las estaciones 2 y 3 , respectivamente.

\section{Composición de especies y abundancia de copépodos}

Las tablas 5 a 7 muestran la presencia de las especies en las diferentes estaciones de recolección. Se registraron 20 especies de copépodos, en total, pertenecientes a 13 géneros y 3 subórdenes mayores: Calanoida (12 especies), Harpacticoida (3) y Cyclopoida (5). Los números máximo y mínimo de copépodos se encontraron en la estación 2, en mayo, y la estación 1, en septiembre, respectivamente. Los géneros Acartia, Acrocalanus y Centropages, pertenecientes a los calanoides, y Euterpina y Oithona, pertenecientes a los harpacticoides y ciclopoides, respectivamente, fueron las formas dominantes. Cuatro especies fueron comunes a las tres estaciones: Acartia southwellii, Centropages orsinii, Euterpina acutifrons y Oithona rigida. Las especies Acartia danae, Oithona similis, Corycaeus catus y Labidocera acuta se encontraron sólo en la zona de mezcla de agua dulce y nerítica.

La abundancia de copépodos mostró variaciones espaciales y temporales. La Fig. 5 presenta la abundancia estacional de copépodos en las tres estaciones de recolección. Del monzón al verano, ésta varió, en promedio, de 2,280 a $46,830 \mathrm{org} . / \mathrm{m}^{3}$ en la estación 1 , de 7,490 a $180,370 \mathrm{org} . / \mathrm{m}^{3}$ en la estación $2 \mathrm{y}$ de 18,930 a 162,180 org. $/ \mathrm{m}^{3}$ en la estación 3. En general, en términos de abundancia numérica, fueron más las especies como Acartia danae, A. southwellii, Acrocalanus longicornis, Pseudodiaptomus serricaudatus, Centropages orsinii, Macrosetella gracilis, Microsetella rosea, Euterpina acutifrons, Oithona rigida, $O$. brevicornis y Corycaeus danae, durante esta observación. 
Table 5. Abundance of copepods at station $1\left(10^{3}\right.$ org. $\left./ \mathrm{m}^{3}\right)$.

Tabla 5. Abundancia de copépodos en la estación $1\left(10^{3} \mathrm{org} . / \mathrm{m}^{3}\right)$.

\begin{tabular}{|c|c|c|c|c|c|c|c|c|c|c|c|c|}
\hline Species & Jan. & Feb. & Mar. & Apr. & May & Jun. & Jul. & Aug. & Sep. & Oct. & Nov. & Dec. \\
\hline \multicolumn{13}{|l|}{ Calanoids } \\
\hline Acartia spinicauda & 0.18 & - & 0.07 & - & 0.35 & - & 0.03 & 1.41 & - & - & - & - \\
\hline Acartia erythraea & - & - & - & - & - & - & - & - & - & - & - & - \\
\hline Acartia danae & - & - & - & - & - & - & - & - & - & - & - & - \\
\hline Acartia southwellii & 0.04 & 0.02 & 0.09 & - & - & - & - & - & 0.03 & 0.61 & - & - \\
\hline Paracalanus parvus & - & - & - & - & - & - & - & - & - & - & - & - \\
\hline Acrocalanus gracilis & 0.06 & 0.02 & - & 0.70 & - & 1.24 & 0.26 & - & - & - & - & - \\
\hline Acrocalanus longicornis & - & - & - & - & - & - & - & - & - & - & - & - \\
\hline Metacalanus aurivillii & - & - & - & - & - & - & - & - & - & - & - & - \\
\hline Centropages orsinii & 0.08 & 0.05 & 0.18 & 0.60 & 0.74 & 1.52 & 2.37 & 3.19 & - & - & - & - \\
\hline Centropages sp. & 0.05 & - & 1.76 & - & - & - & - & - & - & 0.08 & 0.26 & - \\
\hline Pseudodiaptomus serricaudatus & - & - & - & - & - & - & - & - & - & - & - & - \\
\hline Labidocera acuta & - & - & - & - & - & - & - & - & - & - & - & - \\
\hline \multicolumn{13}{|l|}{ Harpacticoids } \\
\hline Macrosetella gracilis & - & - & - & - & - & - & - & - & - & - & - & - \\
\hline Microsetella rosea & 0.39 & 0.19 & 0.68 & 4.67 & 3.42 & 3.43 & - & - & - & $\cdot$ & 0.15 & 0.15 \\
\hline Euterpina acutifrons & 0.18 & 2.24 & 2.84 & - & 4.24 & 0.42 & - & 1.40 & - & - & 0.02 & 0.05 \\
\hline \multicolumn{13}{|l|}{ Cyclopoids } \\
\hline Oithona rigida & 0.03 & 0.50 & 4.50 & 5.38 & 2.96 & 5.42 & 3.58 & 4.34 & - & - & 0.05 & 0.03 \\
\hline Oithona similis & - & - & - & - & - & - & - & - & - & - & - & - \\
\hline Oithona brevicornis & 0.06 & 3.14 & 4.48 & 2.47 & 3.36 & 3.90 & - & - & 0.05 & 0.63 & 0.24 & 0.01 \\
\hline Corycaeus danae & 0.03 & 0.37 & 3.95 & 0.52 & - & 1.49 & 0.17 & 1.40 & - & - & - & - \\
\hline Corycaeus catus & - & - & - & - & - & - & - & - & - & - & - & - \\
\hline Total & 1.1 & 6.53 & 18.55 & 14.34 & 15.07 & 17.42 & 6.41 & 11.74 & 0.08 & 1.32 & 0.72 & 0.24 \\
\hline
\end{tabular}


Table 6. Abundance of copepods at station $2\left(10^{3} \mathrm{org} . / \mathrm{m}^{3}\right)$.

Tabla 6. Abundancia de copépodos en la estación $2\left(10^{3} \mathrm{org} . / \mathrm{m}^{3}\right)$.

\begin{tabular}{|c|c|c|c|c|c|c|c|c|c|c|c|c|}
\hline Species & Jan. & Feb. & Mar. & Apr. & May & Jun. & Jul. & Aug. & Sep. & Oct. & Nov. & Dec. \\
\hline \multicolumn{13}{|l|}{ Calanoids } \\
\hline Acrocalanus gracilis & - & - & - & - & - & - & - & - & 0.59 & - & - & - \\
\hline Acrocalanus longicornis & - & - & - & 0.91 & 6.47 & 0.50 & - & - & $\cdot$ & - & - & - \\
\hline Acartia spinicauda & 0.03 & 0.72 & 3.34 & - & - & - & - & - & - & - & 1.03 & - \\
\hline Acartia erythraea & 0.17 & - & 1.83 & - & - & - & - & - & - & 0.76 & 0.38 & 0.03 \\
\hline Acartia danae & - & - & - & $\cdot$ & 9.32 & 34.34 & 0.54 & 19.75 & $\cdot$ & - & - & - \\
\hline Acartia southwellii & - & - & - & 4.01 & 40.01 & 5.71 & 0.60 & 12.50 & 0.60 & - & - & $\cdot$ \\
\hline Centropages orsinii & 0.69 & 0.45 & - & - & - & - & 0.58 & 0.58 & 0.92 & - & - & - \\
\hline Centropages sp. & - & - & - & - & - & - & - & - & - & - & - & - \\
\hline Labidocera acuta & 0.03 & 0.54 & 0.04 & - & - & - & 0.03 & $\cdot$ & - & 0.18 & 0.82 & 0.73 \\
\hline Metacalanus aurivillii & - & - & - & - & - & - & - & - & - & - & - & - \\
\hline Paracalanus parvus & - & - & 2.62 & 0.22 & - & 0.19 & - & - & 0.63 & - & - & - \\
\hline Pseudodiaptomus serricaudatus & - & - & - & - & - & $\cdot$ & - & - & - & - & - & - \\
\hline \multicolumn{13}{|l|}{ Harpacticoids } \\
\hline Macrosetella gracilis & $\cdot$ & - & 3.35 & - & 4.72 & 0.54 & 3.49 & - & 3.21 & 0.73 & 0.56 & - \\
\hline Microsetella rosea & - & - & - & 1.01 & 1.31 & 28.02 & - & - & 0.25 & - & - & - \\
\hline Euterpina acutifrons & - & - & $\cdot$ & 3.43 & 5.43 & 4.24 & 0.94 & 6.13 & 0.59 & - & - & - \\
\hline \multicolumn{13}{|l|}{ Cyclopoids } \\
\hline Oithona rigida & 1.36 & - & 8.54 & 5.42 & - & 0.07 & 1.03 & 17.41 & 3.47 & 0.39 & 0.17 & 0.13 \\
\hline Oithona similis & - & 3.75 & 0.18 & 0.03 & 13.48 & 1.34 & 0.75 & 2.75 & 7.42 & 0.27 & 0.27 & - \\
\hline Oithona brevicornis & 0.26 & - & 2.43 & 9.24 & - & $\cdot$ & 0.34 & 0.20 & 0.84 & - & - & - \\
\hline Corycaeus danae & - & - & - & - & - & 0.09 & - & - & - & - & 0.05 & 0.32 \\
\hline Corycaeus catus & 0.03 & - & 1.72 & - & - & 0.32 & 0.22 & $\cdot$ & - & 0.19 & 0.90 & 0.03 \\
\hline Total & 2.57 & 5.46 & 24.05 & 24.27 & 80.74 & 75.36 & 8.52 & 59.32 & 18.52 & 2.52 & 4.18 & 1.24 \\
\hline
\end{tabular}


Table 7. Abundance of copepods at station $3\left(10^{3} \mathrm{org} . / \mathrm{m}^{3}\right)$

Tabla 7. Abundancia de copépodos en la estación $3\left(10^{3} \mathrm{org} . / \mathrm{m}^{3}\right)$.

\begin{tabular}{|c|c|c|c|c|c|c|c|c|c|c|c|c|}
\hline Species & Jan. & Feb. & Mar. & Apr. & May & Jun. & Jul. & Aug. & Sep. & Oct. & Nov. & Dec. \\
\hline \multicolumn{13}{|l|}{ Calanoids } \\
\hline Acartia erythraea & 1.06 & - & 11.30 & - & 17.39 & - & 0.32 & 28.40 & 1.21 & - & 3.36 & 0.02 \\
\hline Acartia spinicauda & - & - & - & - & - & - & - & - & - & - & - & - \\
\hline Acartia danae & - & - & - & - & - & - & - & $\cdot$ & - & - & - & $\cdot$ \\
\hline Acartia southwellii & 0.17 & 1.02 & - & 25.95 & - & 11.22 & 7.67 & 0.26 & - & 1.61 & - & - \\
\hline Paracalanus parvus & - & - & - & 0.95 & - & - & 0.69 & 0.46 & 0.05 & - & 0.03 & 0.02 \\
\hline Acrocalanus gracilis & - & - & - & - & - & - & - & - & - & - & - & - \\
\hline Acrocalanus longicornis & 0.49 & 0.70 & 0.14 & - & - & 22.36 & 2.27 & 0.33 & 0.02 & - & - & 0.07 \\
\hline Metacalanus aurivillii & 0.09 & 0.23 & 0.59 & 1.09 & - & - & 0.55 & 0.07 & - & - & 0.01 & - \\
\hline Centropages orsinii & 0.34 & - & 0.69 & - & $=$ & - & 1.09 & 0.07 & 0.69 & - & - & 0.06 \\
\hline Centropages sp. & 0.02 & 8.11 & 0.35 & - & - & - & - & - & - & 1.33 & 5.75 & 0.33 \\
\hline Pseudodiaptomus serricaudatus & 0.47 & 2.32 & 3.21 & 4.72 & - & 12.75 & 2.94 & 3.42 & 0.27 & - & - & - \\
\hline Labidocera acuta & - & - & - & - & - & - & - & - & - & - & - & - \\
\hline \multicolumn{13}{|l|}{ Harpacticoids } \\
\hline Macrosetella gracilis & - & 0.22 & 2.75 & 0.55 & 13.81 & - & 5.44 & 5.34 & - & - & 0.33 & - \\
\hline Microsetella rosea & - & - & - & - & - & - & - & - & - & - & - & - \\
\hline Euterpina acutifrons & 0.17 & 0.75 & 8.43 & 5.43 & 6.94 & 0.19 & 6.48 & 11.02 & 16.97 & - & 0.03 & - \\
\hline \multicolumn{13}{|l|}{ Cyclopoids } \\
\hline Oithona rigida & 1.53 & 0.07 & 2.78 & 6.46 & 8.54 & 23.83 & 9.47 & 19.16 & 0.12 & 2.41 & 0.54 & 3.03 \\
\hline Oithona similis & - & - & - & - & - & - & - & - & - & - & - & - \\
\hline Oithona brevicornis & - & - & - & - & - & - & - & - & - & - & - & - \\
\hline Corycaeus danae & - & - & - & - & - & - & - & - & - & - & - & - \\
\hline Corycaeus catus & - & - & - & - & - & - & - & - & - & - & - & - \\
\hline Total & 4.34 & 13.42 & 30.24 & 45.15 & 46.68 & 70.35 & 36.92 & 68.53 & 19.33 & 5.35 & 10.05 & 3.53 \\
\hline
\end{tabular}




\section{DISCUSSION}

The variable nature of the environmental parameters would affect the occurrence, composition and distribution of the plankton. Among the various zooplankton, tintinnids and copepods were the major components throughout the study period, as has been reported for different Indian mangroves: $91 \%$ by Subbaraju and Krishnamurthy (1972), $71.75 \%$ by Goswami and Selvakumar (1977), $89.75 \%$ by Damodara Naidu (1980), $89.30 \%$ by Bhunia and Choudhury (1981). It would appear that the species composition and abundance of tintinnids and copepods in the Pichavaram mangroves were rather poor when compared to the adjacent biotopes such as Vellar estuary or the coastal sea [up to 70 species by Subbaraju (1968); 49 species by Damodara Naidu (1980); 45 species by Baskaran (1984); 130 species by Krishnaswamy (1953)].

The occurrence of only a few species in the waterways of the mangrove ecosystem could be due to the shallowness of the water column, the varying water salinity because of the constant ebb and flow of tides and the highly turbid nature of the water. Dutta et al. (1954) and Baidya (1984) reported in Hooghly estuary of West Bengal, that the turbidity of water plays an important role in the distribution of zooplankton. Higher occurrence and abundance of species at stations 2 and 3 might be due to the migration of neritic species into this area during high tide. The higher values of temperature and salinity recorded may also contribute to the greater number of species found at these stations. The lower number at station 1 might be due to the frequent inflow of freshwater from the adjacent irrigation channels. The input of freshwater into sation 1 from the upland regions brings considerable amounts of terrigenous material and accounts for the highly turbid nature of the water at this station. This could have been the reason for the lower number of tintinnids and copepods recorded at this station throughout the year. Damodara Naidu (1980) and Palaniappan and Baskaran (1985) also observed a greater number of zooplanktonic species in the interior of the mangroves. The following reasons may be attributed to the recorded maximum abun-
Las especies Acartia southwellii, A. danae, Acrocalanus longicornis, Macrosetella gracilis, Euterpina acutifrons y Oithona similis fueron las responsables de esta máxima abundancia. Durante la abundancia mínima, únicamente se encontraron Acartia southwellii y Oithona similis.

\section{Coeficiente de correlación}

En las tres estaciones, se observó una correlación positiva notable entre la abundancia mensual de copépodos y tintínidos. La tabla 8 muestra una correlación altamente significativa entre la abundancia mensual de tintínidos y la de copépodos, en las tres estaciones. Esta puede mostrar la relación de su red alimentaria en este ecosistema.

\section{DISCUSION}

La naturaleza variable de los parámetros ambientales puede afectar la presencia, composición y distribución del plancton. De la variedad del zooplancton, tintínidos y copépodos fueron los mayores componentes durante el periodo de estudio, como sucede en diferentes manglares de la India según informan: Subbaraju y Krishnamurthy (1972), 91\%; Goswami y Selvakumar (1977), 71.75\%; Damodara Naidu (1980) 89.75\%; Bhunia y Choudhury (1981), $89.30 \%$. Parece ser que la composición de especies y la abundancia de tintínidos y copépodos en los manglares de Pichavaram fue más bien pobre si se compara con los biotopos adyacentes, como el estuario Vellar o el mar costero [hasta 70 especies según Subbaraju (1968); 49, según Damodara Naidu (1980); 45, según Baskaran (1984); 130, según Krishnaswamy (1953)].

La presencia de sólo algunas especies en los canales del ecosistema de manglares podía deberse a la poca profundidad de la columna de agua, la salinidad variante del agua por el constante flujo y reflujo de las mareas y la naturaleza altamente turbia del agua. Dutta et al. (1954) y Baidya (1984) informaron que, en el estuario Hooghly de Bengala Occidental, la turbidez del agua tiene un papel importante en la distribución del zooplancton. La mayor pre- 
Table 8. Simple correlation coefficient ( $r$ ) values and probability values between the monthly abundance of copepods and tintinnids.

Tabla 8. Valores del coeficiente de correlación simple (r) y valores de probabilidad entre la abundancia mensual de copépodos y tintínidos.

\begin{tabular}{ccc}
\hline Station & Correlation coefficient $(r)$ & Probability value \\
\hline 1 & 0.8626 & $\mathbf{P}<0.001$ \\
2 & 0.7770 & $\mathbf{P}<0.01$ \\
3 & 0.9274 & $\mathrm{P}<0.001$ \\
\hline
\end{tabular}

dance of both groups during summer: (a) for tintinnids the regularity of the reproductive cycle and rapid cell division; (b) for copepods to breed, propagate and colonize.

During summer, a greater number of smaller-sized tintinnids were observed. This could be due to the relative consistency of temperature and salinity regimes of the water column. The dominance of smaller-sized food items like naked flagellates, smaller-sized diatoms and peridinians during this season could also be the reason for their peak abundance. Similar observations were made by Tranter and Abraham (1971), Subbaraju and Krishnamurthy (1972), Krishnamurthy and Santhanam (1975), Damodara Naidu (1980). This observation of smaller-sized diatoms, dinoflagellates and peridinians in these mangrove waters is similar to the observation of Mani (1989), who estimated total phytoplankton biomass $\left(9.99 \times 10^{5}\right.$ cells $\left./ l\right)$ with dominance of smaller-sized phytoplankton during summer. Kannan and Krishnamurthy (1979) also estimated the biomass of photosynthetic bacterioplankton (bacteriochlorophyl] $30.23 \mathrm{mg} / \mathrm{m}^{3}$ ) and phytoplankton (chlorophyll $a$ $24.57 \mathrm{mg} / \mathrm{m}^{3}$ ) in these mangrove waters.

For tintinnids, the species of the genus Tintinnopsis were the most important in terms of the number of species. Species like Tintinnopsis cylindrica, T. mortensenil, Favella philippinensis and $F$. brevis contributed to the maximum abundance. This may be due to the high reproductive capacity of the species. Similarly, Damodara Naidu (1980) and Jegadeesan (1986) also noticed such tintinnid species dominancy in the bulk of the biomass of microzooplankton in the mangrove waters and adjacent Coleroon estuarine complex. sencia y abundancia de especies en las extaciones 2 y 3 puede deberse a la inmigración de las especies neriticas a esta zona durante la marea alla. Los altos valores de lemperatura y salinidad registrados pueden tambićn contribuit al alto número de especies encontrado en estas estaciones. El número menor encontrado en la estación 1 puede deberse al frecuente flujo entrante de agua dulce de los canales adyacentes de irrigación. La entrada de agua dulce proveniente de las regiones altas lleva a la estación lcantidades considerables de material terrígeno y causa la naturaleza altamente turbia del agua. Esta puede haber sido la razón de que fuera menor el número de tintínidos y copépodos registrados en esta estación durante todo el año. Damodara Naidu (1980), y Palaniappan y Baskaran (1985) también observaron un número mayor de especies zooplanctónicas en cl interior de los manglares. La máxima abundancia registrada de ambos grupos durante el verano se puede atribuir a las siguientes razones: a) en el caso de los tintínidos, la regularidad del ciclo reproductivo y la rápida división celular; b) en el caso de los copépodos, la crianza, propagación y colonización.

Durante el verano, se observó un número mayor de tintínidos de menor tamaño, probablemente debido a la relativa consistencia de los regimenes de temperatura y salinidad de la columna de agua. Durante esta estación, el dominio de elementos alimenticios de menor tamaño, como flagelados descubicrtos, diatomcas menores y perideos, podría también ser la razón de su pico de abundancia. Tranter y Abraham (1971), Subbaraju y Krishnamurthy (1972), Krishnamurthy y Santhanam (1975) y Damodara Naidu (1980) han hecho observaciones simi- 
Among the non-agglomerated tintinnids, Favella philippinensis and $F$. brevis were the dominant forms during the peak of tintinnid abundance. This might be due to the rapid cell division of the species and the abundance of suitable food items. A swarm of $F$. philippinensis $\left(12,858,000 \mathrm{org} . / \mathrm{m}^{3}\right)$ was also recorded in the Vellar estuary by Krishnamurthy and Damodara Naidu (1977).

Among the species of copepods, the calanoids were represented by more species than the cyclopoids. The calanoid Acartia spp. and cyclopoid Oithona spp. were found to be dominant in the present study. This may be due to their continuous breeding behaviour, quick larval development and that they adopt well to the environmental conditions of the mangroves. This dominance of Acartia spp. among calanoids was reported for the Godavari estuary (Chandramohan, 1963) and Cochin backwaters (Menon et al., 1971; Madhupratap, 1978), and that of Oithona spp. among cyclopoids was noticed in the Vellar estuary (Chandran, 1982; Sivakumar, 1982). Similar findings were also noticed in Malaysian waters by Chong and Chua (1973), who pointed out that the abundance of Oithona spp. was mainly due to its high reproductive capacity.

The dominant copepods in the present study were Acartia danae, A. southwellii, Acrocalanus gracilis, Pseudodiaptomus serricaudatus, Euterpina acutifrons, Macrosetella gracilis, Microsetella rosea, Oithona rigida and Oithona similis. The abundance of these species might be due to their continuous breeding nature and the suitable environmental conditions of the ecosystem. Similar dominance of this species in bulk to the biomass of macrozooplankton in the Mandovi and Zuari estuaries was also recorded by Dwivedi et al. (1974) and in the Vellar estuary by Thangaraj (1984).

The maximum monthly abundance of tintinnids coincided with the maximum monthly copepod abundance. The correlation coefficient and probability values showed a significant relationship between the abundance of tintinnids and copepods. This could be attributed to the trophic relationship existing between tintinnids and copepods. The peak of copepod abundance $\left(80,740\right.$ org. $\left./ \mathrm{m}^{3}\right)$ during summer was succeeded by the peak of tintinnid abun- lares. Las observaciones de diatomeas menores, dinoflagelados y perideos efectuadas en las aguas de estos manglares son similares a las de Mani (1989), quien estimó la biomasa total del fitoplancton, $9.99 \times 10^{5} \mathrm{cel} / 1$, con dominio del fitoplancton de tamaño más pequeño, durante el verano. Kannan y Krishnamurthy (1979) también estimaron la biomasa del bacterioplancton fotosintético (bacterioclorofila $30.23 \mathrm{mg} / \mathrm{m}^{3}$ ), y fitoplancton (clorofila a $24.57 \mathrm{mg} / \mathrm{m}^{3}$ ), en las aguas de estos manglares.

Para los tintínidos, las especies del género Tintinnopsis fueron las más importantes, en términos del número de especies. Las especies como Tintinnopsis cylindrica, $T$. mortensenii. Favella philippinensis y $F$. brevis contribuyeron a la abundancia máxima. Esto puede deberse a la alta capacidad reproductiva de las especies. De manera parecida, Damodara Naidu (1980) y Jegadeesan (1986) notaron este dominio de las especies de tintínidos en la mayor parte de la biomasa del microzooplancton, en las aguas de los manglares y en el complejo estuarino adyacente de Coleroon.

Entre los tintínidos no aglomerados, Favella philippinensis y $F$. brevis fueron las formas dominantes durante el pico de abundancia de tintínidos. Esto puede deberse a la rápida división celular de las especies y la abundancia de elementos alimenticios apropiados. Una multitud de $F$. philippineisis $\left(12,850,000\right.$ org. $\left./ \mathrm{m}^{3}\right)$ fue también registrada por Krishnamurthy y Damodara Naidu (1977), en el estuario Vellar.

Entre las especies de copépodos, los calanoides estuvieron representados por más especies que los ciclopoides. Se encontró que el calanoide Acartia spp. y el ciclopoide Oithona spp. fueron dominantes en el presente estudio. Esto puede deberse a su comportamiento reproductivo continuo, desarrollo larval rápido y a que se adaptan bien a las cambiantes condiciones ambientales de los manglares. Este dominio de Acartia spp. entre los calanoides fue observado en el estuario de Godavari (Chandramohan, 1963), el remanso de Cochin (Menon et al., 1971; Madhupratap, 1978), y el de Oithona spp. entre los ciclopoides fue observado en el estuario de Vellar (Chadran, 1982; Sivakumar, 1982). Chong y Chua (1973) encontraron proporciones similares en aguas malasias y señalaron que la abundancia de $\mathrm{O} i$ - 
dance $\left(44,990 \mathrm{org} . / \mathrm{m}^{3}\right)$ during the tail end of summer and the early premonsoon season. Damodara Naidu (1980) also observed such a significant relationship between these two groups in mangrove waters during his investigation.

The low abundance of plankton recorded during the monsoon season might be due to the non-availability of food, low temperature and low salinity. The disturbance of the food web and minimum production of plankton during the monsoon season have been observed in many Indian estuaries and mangroves (Dutta $e t$ al., 1954; Shetty et al., 1982; Subbaraju and Krishnamurthy, 1972; Madhupratap, 1976; Damodara Naidu, 1980; Prince Jeyaseelan and Krishnamurthy, 1980; Bhunia and Choudhury, 1981). During the monsoon, the values of salinity decreased ( 3.4 and $18.7 \mathrm{ppt}$ ) owing to the inflow of freshwater. The low salinity would drastically affect the plankton abundance (Wilson, 1942; Damodara Naidu, 1980; Baskaran, 1984). In the present investigation, the increase or decrease of salinity in the water column exert either a direct or an indirect effect on species composition and abundance. A direct effect is the disappearance of some forms and replacement by others. The second effect is probably the migration of some species from one station to another to avoid either low or high salinity. The indirect effect might be due to the scarcity of food caused by the fluctuations of salinity in the waters, ultimately affecting the population abundance of tintinnids and conepods.

\section{ACKNOWLEDGEMENTS}

The author thanks K. Krishnamurthy (C.A.S. in Marine Biology, Annamalai University) for his constant encouragement and guidance, and the Ministry of Environment \& Forests, Government of India, for financial assistance to carry out this work. I hanks are also due to the Director of C.A.S. in Marine Biology for the facilities provided. The partial financial support extended to the author by the organizers of the Fourth International Conference on Copepoda, Japan, and the Tamil Nadu State Council for Science \& Technology to participate in the conference, where a brief ver- thona spp. se debió principalmente a su alta capacidad reproductora.

Los copépodos dominantes en el presente estudio fueron Acartia danae. A. southwellit. Acrocalanus gracilis, Pseudodiaptomus serricaudatus, Euterpina acutifrons, Macrosetella gracilis, Microsetella rosea, Oithona rigida y Oithona similis. La abundancia de estas especies puede deberse a su naturaleza de reproducción continua y a condicioncs ambientales apropiadas del ecosistema. Un dominio similar de esta especie en la mayoria de la biomasa del macrozooplancton fue registrado por Dwivedi et al.(1974), en los estuarios de Mandovi y Zuari, y por Thangaraj (1984), en el estuario de Vellar.

La abundancia máxima mensual de tintínidos coincide con la abundancia máxima mensual de copépodos. Los valores del coeficiente de correlación (r) y de probabilidad muestran una relación significativa entre la abundancia de tintínidos y copépodos. Esto puede atribuise a la relación trófica existente entre ambos. El pico de la abundancia de copépodos durante el verano $\left(80,740 \mathrm{org} . / \mathrm{m}^{3}\right)$ fue seguido por el pico de la de tintínidos $\left(44,990 \mathrm{org} . / \mathrm{m}^{3}\right)$ durante el final del verano y el inicio de la estación premonzónica. Damodara Naidu (1980) también observó esta relación significativa entre los dos grupos, en aguas de manglares.

La baja abundancia de plancton registrada durante la estación del monzón puede deberse a la falta de disponibilidad de alimento, así como a bajas temperatura y salinidad. La perturbación de la cadena alimentaria y la mínima producción de plancton durante la estación del monzón se observó en muchos estuarios y manglares de la India (Dutta et al., 1954; Shetty et al., 1982; Subbaraju y Krishnamurthy, 1972; Madhupratap, 1976; Damodara Naidu, 1980; Prince Jeyaseelan y Krishnamurthy, 1980; Bhunia y Choudhury, 1981). Durante el monzón, los valores de salinidad disminuyeron $(3.4 \mathrm{y}$ $18.7 \mathrm{ppm}$ ) debido a la entrada de agua dulce. La baja salinidad afecta drásticamente la abundancia de plancton (Wilson, 1942; Damodara Naidu, 1980; Baskaran, 1984). En la presente investigación, el aumento o disminución de la salinidad en la columna de agua ejerció un efecto directo o indirecto sobre la composición y abundancia de especies. Un 
sion of the paper was presented, is gratefully acknowledged.

\section{REFERENCES}

Baidya, A.U. (1984). Ecology of the Copepoda (Calanoid) components of zooplankton in Hooghly estuary, West Bengal, India. Ph.D. thesis, Calcutta University, 92 pp.

Baskaran, K. (1984). Studies in estuarine and backwater copepods. M.Phil. thesis, Annamalai University, $116 \mathrm{pp}$.

Beers, J.R. and Stewart, G.L. (1969). Microzooplankton and its abundance relative to the larger zooplankton and other seston components. Mar. Biol., 4: 182-189.

Bhunia, A.B. and Choudhury, A. (1981). Studies on the seasonal abundance and biomass of crustacean zooplankton and chaetognaths in relation to ecological parameters of a tidal creek (Mooriganga) of Sagar island (North), Sunderbans, West Bengal. Proc. Symp. Ecol. Anim. Popul. Zool. Surv., India, pp. 175-183.

Capriulo, G.M. and Carpenter, E.J. (1983). Abundance, species composition and feeding impact of tintinnid microzooplankton in central Long Island Sound. Mar. Ecol. Prog. Ser., 10: 277-288.

Chandran, R. (1982). Hydrobiological studies in the gradient zone of the Vellar estuary. $\mathrm{Ph} . \mathrm{D}$. thesis, Annamalai University, 195 pp.

Chandramohan, P. (1982). Studies on zooplankton of the Godavari estuary. Ph.D. thesis, Annamalai University, $163 \mathrm{pp}$.

Chong, B.J. and Chua, T.D. (1973). A preliminary study of the distribution of the cyclopoid copepods of the family Oithonidae in Malaysian waters. Pacific Science Association Special Symposium on Marine Science, pp. 32-36.

Conover, R.J. (1982). Interrelation between microzooplankton and other plankton organisms. Annls. Inst. Oceanogr., Paris, 58(5): 31-46.

Damodara Naidu, W. (1980). Studies on tintinnids (Protozoa: Ciliata) of Porto Novo region, South India. Ph.D. thesis, Annamalai University, $281 \mathrm{pp}$. efecto directo es la desaparición de algunas formas y la sustitución por otras. El segundo efecto es probablemente la emigración de ciertas especies de una estación a otra, para evitar la baja o alta salinidad. El efecto indirecto puede deberse a la escasez de alimento causada por las fructuaciones de salinidad en las aguas, que $\mathrm{fi}_{i}$ nalmente afecta a la abundancia de la población de tintínidos y copépodos.

\section{AGRADECIMIENTOS}

El autor agradece a K. Krishnamurthy, del Centro de Estudios Avanzados en Biología Marina, Universidad Annamalai, su constante estímulo y guía, y al Ministro del Ambiente y Bosques, del Gobierno de la India, la asistencia financiera para llevar a cabo este trabajo. Se agradecen también las facilidades proporcionadas por el Director del centro de investigación mencionado. Se reconoce ampliamente el financiamiento parcial dado al autor por los organizadores de la Cuarta Conferencia Internacional sobre Copepoda, Japón, y el Consejo Estatal de Tamil Nadu para la Ciencia y la Tecnología, a fin de participar en la conferencia, donde se presentó una versión breve del artículo. Mora

Traducido al español por Olivia Gómez

Damodara Naidu, W., Santhanam, R., Krishnamurthy, K. and Natarajan, R. (1977). The species biomass and the seasonal composition of tintinnids (Protozoa: Ciliata). Proc. Symp. Warm Water Zool. Spl. Publ. UNESCO/NIO, pp. 520-527.

Dussart, B.H. (1965). Les différentes catégories du plancton. Hidrobiologia, 26: 72-74.

Dutta, N., Malhotra, J.C. and Bose, B.B (1954). Hydrology and seasonal fluctuation of the plankton in Hooghly estuary. Symp. Mar. Freshwat. Plankton in the Indo-Pacific IPFC, Bangkok, pp. 35-47.

Dwivedi, S.N., Bhargava, R.M.S., Parulekar, A.H., Selvakumar, R.A., Singbal, S.Y.S. and Sankaranarayanan, V.N. (1974). Ecology and environmental monitoring of Mandovi, Zuari and Comburzua canal 
complex during monsoon months. J. Indian Fish. Ass., $3 \& 4(1 \& 2): 113-130$.

Goswami, S.C. and Selvakumar, R.A. (1977). Plankton studies in the estuarine system of Goa. Proc. Symp. Warm Water Zoopl. Spt. Publ. UNESCO/NIO, pp. 228-241.

Jegadeesan, P. (1986). Studies on environmental inventory of the marine zone of Coleroon estuary and inshore waters of Pazhayaru, southeast coast of India. Ph.D. thesis, Annamalai University, $277 \mathrm{pp}$.

Kanna, L. and Krishnamurthy, K. (1979). Biomass of photosynthetic bacterioplankton and phytoplankton. Indian J. Microbiol., 19(2): 64-68.

Krishnamurthy, K. (1962). Daily variations in marine plankton from Porto Novo, S. India. J. Zool. Soc. India, 13: 180-187.

Krishnamurthy, K. and Santhanam, R. (1975). Ecology of tintinnids (Protozoa: Ciliata) in Porto Novo region. Indian J. Mar. Sci., 4: 181-184.

Krishnamurthy, K. and Damodara Naidu, W. (1977). Swarming of the tintinnids (Protozoa: Ciliata) in the Vellar estuary. Curr. Sci., $46(11): 384$.

Krishnamurthy, K., Santhanam, R. and Sundararaj, V. (1979). The trophic tier. Marine Sciences, 209-217.

Krishnaswamy, S. (1953). Pelagic copepods of the Madras coast. J. Madras Univ. B., 23: 67-75, 107-144.

Madhupratap, M. (1976). Studies on the ecology of zooplankton of Cochin backwaters (a tropical estuary). Ph.D. thesis, Cochin University.

Madhupratap, M. (1978). Studies on the ecology of zooplankton of Cochin backwaters. Mahasagar Bull. Nat. Inst. Oceanogr., 1 (1\&2): 45-46.

Mani, P. (1989). Phytoplankton ccology of the mangrove ecosystems. Ph.D. thesis, Annamalai University, $122 \mathrm{pp}$.

Menon, N.R., Venugopal, P. and Goswami, S.C. (1971). Total biomass and faunistic composition of zooplankton in the Cochin backwaters. J. Mar. Biol. Ass. India, 13: 220-225.
Palaniappan, R. and Baskaran, K. (1985). Distribution and abundance of zooplankton in Pichavaram mangrove, South India. The mangroves. Proc. Nat. Symp. Biol. Util. Cons. Mangroves, pp. 499-504.

Prince Jeyaseelan, M.J. and Krishnamurthy, K. (1980). Role of mangrove forests of Pichavaram as fish nurseries. Proc. Indian Natn. Sci. Acad. B., 46: 48-53.

Shetty, H.P.C., Saha, S.B. and Ghosh, B.B. (1982). Observation on the distribution and fluctuations of plankton in the Hooghly-Maltah estuary system, with notes on their relation to commercial fish landings. Indian J. Fish., 8: 326-363.

Shukhanova, Z.N. (1978). Settling without the inverted microscope. In: A. Sournia (ed.), Phytoplankton Manual UNESCO, Page Brothers (Noruie) Ltd., $97 \mathrm{pp}$.

Sivakumar, V. (1982). An environmental inventory of the tidal zone of the Vellar estuary. Ph.D. thesis, Annamalai University, $178 \mathrm{pp}$.

Strickland, J.D.H. and Parsons, T.R. (1972). A practical handbook of seawater analysis. Bull. Fish. Res. Bd. Canada, 167: 310.

Subbaraju, R.C. (1968). Studies on planktonic copepods of estuarine and inshore waters at Porto Novo (S. India). Ph.D. thesis, Annamāai Ũniversity.

Subbaraju, R.C. and Krishnamurthy, K. (1972). Ecological aspects of plankton production. Mar. Biol., 14: 25-31.

Sundararaj, V. and Krishnamurthy, K. (1974). Studies on phytoplankton pigments in Porto Novo waters (India). I. Mangrove. J. Exp. Mar. Biol. Ecol., 14: 275-284.

Thangaraj, G.S. (1984). Ecobiology of the marine zone of the Vellar estuary. Ph.D. thesis, Annamalai University, $192 \mathrm{pp}$.

Tranter, D.J. and Abraham, A. (1971). Cocxistence of species of Acartiidae (Copepoda) in the Cochin backwaters, a monsoonal estuarine lagoon. Mar. Biol., 11: 222-241.

Utermöhl, H. (1958). Zur Vervollkommnung der quantitativen phytoplankton methodik. Mitt. Int. Verein. Theor. Angew. Limnol., 9: 38 .

Wilson, C.B. (1942). Carnegie Inst. Wash. Pub., 536. 